\title{
Reconfiguration of charge density waves by surface nanostructures on $\mathrm{TaS}_{2}$
}

\author{
R. Adelung, J. Brandt, L. Kipp, and M. Skibowski \\ Institut für Experimentelle und Angewandte Physik, Universität Kiel, D-24098 Kiel, Germany
}

(Received 22 September 2000; published 6 April 2001)

\begin{abstract}
The transition metal dichalcogenide $1 T-\mathrm{TaS}_{2}$ forms charge density waves due to the quasi-twodimensionality of its electronic structure. Here we show how the growth of Rb nanowires on the surface of $1 T-\mathrm{TaS}_{2}$ affects the superstructure of the charge density waves. Scanning tunneling microscopy and lowenergy electron diffraction studies show the replacement of the normal $\mathrm{TaS}_{2}$ room temperature $\sqrt{13} \times \sqrt{13}$ phase by $c(2 \sqrt{3} \times 4)$ rect. and $3 \times 3$ superstructures depending on the state of nanowire growth. Furthermore, angle resolved photoemission studies give evidence for a metal to semiconductor transition when the superstructure of the charge density wave is changed. These changes are discussed in the context of nanowire growth, which is accompanied by a change in the dimensionality of the electronic states of $1 T-\mathrm{TaS}_{2}$.
\end{abstract}

DOI: 10.1103/PhysRevB.63.165327

PACS number(s): 79.60.Jv, 68.37.Ef, 64.60.-i

\section{INTRODUCTION}

The geometric and electronic structures of layered transition metal dichalcogenides (TMDC's) have been the subject of many experimental and theoretical investigations (see, e.g., Refs. 1-6). This is mainly due to their quasi-twodimensionality and resulting unique physical properties, exhibiting phase transitions and charge density waves (CDW's). As model systems for high-temperature superconducting cuprates their enhanced electron-phonon interactions have led to increased interest in TMDC's recently. ${ }^{7-9}$ Depending on temperature the periodicity of the CDW's is subject to a series of phase transitions with considerable influence on the electronic structure in the vicinity of the Fermi level. For $1 T-\mathrm{TaS}_{2}$ there is a metal-semiconductor transition at around $180 \mathrm{~K}$ associated with a structural transition due to electron-phonon coupling from a nearly commensurate (NC) to a commensurate (C) CDW phase accompanied by a completely locked-in $\sqrt{13} \times \sqrt{13}$ superstructure. In this phase transition a gap opens at the Fermi level. ${ }^{1,10-13}$ For $1 T-\mathrm{TaS}_{2}$ it is well accepted that this mechanism is driven by the existence of large parallel sheets of the Fermi surface (Fermi vector nesting) resulting in an enhanced dielectric susceptibility. ${ }^{14}$ Compared to the similar Peierls instability in one-dimensional metals, ${ }^{15,16}$ in quasi-two-dimensional $1 T-\mathrm{TaS}_{2}$ only fractions of the Fermi surface satisfy the nesting condition.

It is well known that (concerning their phase angle) charge density waves in layered materials are pinned by defects. ${ }^{16}$ Complete structural changes of the charge density waves, however, can also be obtained by electrochemical intercalation. $^{17,18}$ In particular, electrochemical or vacuum intercalation of guest materials into the van der Waals gap between the sandwich layers results in different periodicities of the CDW's. Superstructures revealing $3 \times 3$ or the so called $\sqrt{7} / 8 \times \sqrt{7} / 8$ structure are often observed and seem to be substantially independent of the type of intercalated material. ${ }^{19,20}$ All these intercalation processes have in common an increase in the layer perpendicular lattice parameter $c,{ }^{21,32}$ resulting in an enhanced two-dimensionality of electronic states. Consequently, larger parallel sheets of the Fermi surface perpendicular to the layers are obtained, lead- ing to pronounced changes of the properties of the associated CDW's. It should be noted here, however, that upon electrochemical intercalation the lattice parameter $c$ can only be increased in discrete steps depending on the size of the intercalated material. Furthermore, due to a significant change of the chemical composition of the intercalated material a strong influence on the electronic structure of the intercalation compound itself is expected.

It has been shown recently that exposure of alkali-metal atoms to surfaces of layered transition metal dichalcogenides in ultrahigh vacuum results in growth of nanowires which finally form networks on the surfaces. ${ }^{23}$ Typical total coverage of the surface with nanowires is below $4 \%{ }^{24}$ and average diameters of the layered material inside the meshes are of the order of $1 \mu \mathrm{m}$. Associated with the formation of the nanowire network on the surface is a corrugation of the surface layer combined with a slight elevation depending on the state of network growth. This elevation of the surface layer, which can be continuously tuned by the amount of nanowires grown on the surface, leads to an electronic decoupling of the surface layer electronic states from the bulk states. Thereby the dimensionality of the electronic states and the fraction of parallel Fermi surface sheets can be continuously adjusted. It should be noted here that the adjustment of dimensionality is achieved by maintaining the chemical composition of the layered crystal between the nanowires, which contrasts with intercalation studies where the chemical composition is drastically changed.

In this paper we show how the growth of $\mathrm{Rb}$ nanowires on the layered material $1 T-\mathrm{TaS}_{2}$ can be used to manipulate the CDW's and associated electronic structure.

\section{EXPERIMENT}

All images shown here were taken with a standard scanning tunneling microscope (STM). ${ }^{25}$ Samples can be moved in vacuo between interconnected pods containing the preparation system (base pressure below $10^{-10} \mathrm{mbar}$ ), an angle resolved photoemission spectrometer, and a low-energy electron diffraction (LEED) system. Angle resolved photoemission spectra were taken either with $\mathrm{He} I_{\alpha}$ radiation $(21.2 \mathrm{eV}$ photon energy) or with synchrotron radiation supplied from 

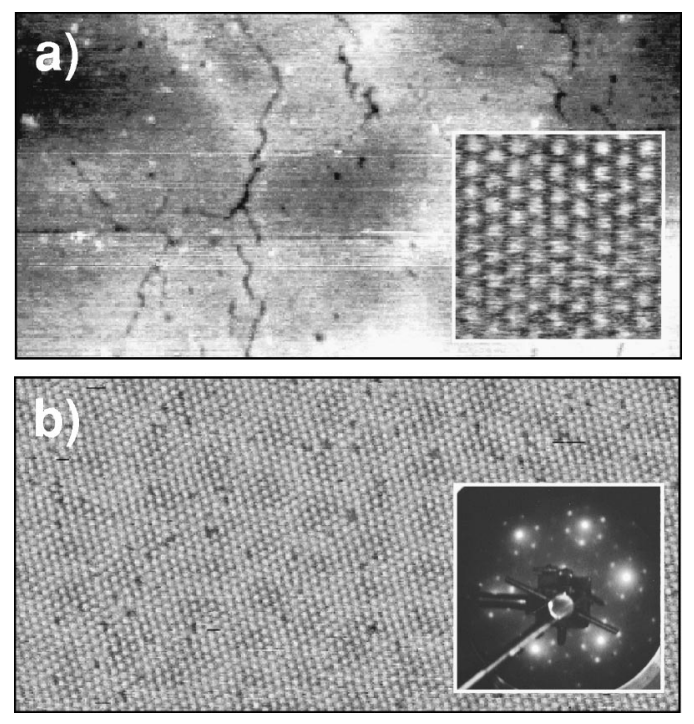

FIG. 1. Room temperature STM empty state (bias $0.7 \mathrm{eV}, I_{T}$ $=200 \mathrm{pA}$ ) images of $1 T-\mathrm{TaS}_{2}$ surfaces. Horizontal dimensions are $120 \mathrm{~nm}$. (a) Quenched crystal surface exhibiting large cracks. The inset shows the CDW superstructure in an enlarged area between the cracks. (b) More slowly cooled ( $\sim 25 \mathrm{~K} / \mathrm{min})$ sample showing proper CDW's without cracks. Both surfaces provide a similar LEED pattern as shown in the inset.

the BW3 $(h \nu=30-80 \mathrm{eV})$ and HONORMI $(8-30 \mathrm{eV})$ beamlines using the spectrometer ASPHERE at HASYLAB in Hamburg. ${ }^{26}$ An angular resolution of less than $1^{\circ}$ and an energy resolution of $\Delta E=75 \mathrm{meV}$ were chosen.

\section{SAMPLE PREPARATION AND NANOWIRE GROWTH}

All results shown here were obtained from chemical vapor transport (CVT) grown $\mathrm{TaS}_{2}$ samples using iodine as transport agent. Clean surfaces were prepared by cleavage in ultrahigh vacuum. Depending on the cooling procedure after CVT growth, $1 \mathrm{~T}$ or $2 \mathrm{H}$ polytypes of $\mathrm{TaS}_{2}$ can be formed. In general quenching (i.e., shock cooling of the crystals to room temperature) right after growth is performed to obtain the $1 T$ phase. An alternative to get the $1 T$ phase is described in Ref. 5 , where $\mathrm{ICl}_{2}$ is used as transport. Extreme quenching, however, often results in poor crystal quality. Using scanning tunneling microscopy we have characterized cleaved surfaces of $\mathrm{TaS}_{2}$ samples that have been quenched and more slowly cooled ( $\sim 25 \mathrm{~K} / \mathrm{min})$ after growth, respectively.

In Fig. 1 we show STM images for a quenched [1(a)] and a more slowly cooled [1(b)] $\mathrm{TaS}_{2}$ sample. While the quenched samples show a number of cracks with typical depths of more than $20 \mathrm{~nm}$, the more slowly cooled crystals exhibit no major defects on the same length scale and the corrugations due to the charge density waves characterizing the $1 T$ phase are clearly visible. Interestingly, both surfaces provide relatively sharp LEED images revealing the same CDW superstructures [see inset in Fig. 1(b)]. It is evident that quenched samples are not suitable for adsorption or intercalation experiments because even large molecules can easily diffuse along cracks into the bulk of the material. For all adsorption experiments shown in this work we used more slowly cooled $1 T-\mathrm{TaS}_{2}$ substrates exhibiting proper charge density waves in the NC phase at room temperature.

$\mathrm{Rb}$ exposure was performed using commercial SAES Getter sources. ${ }^{27}$ The $1 T-\mathrm{TaS}_{2}$ substrates were kept at room temperature. Depending on the dispenser current two different evaporation modes were applied. Evaporation at typical currents of 6-7 A results in a glowing of the dispenser. This will be described in the following as the "hot" evaporation mode to accentuate that the material is evaporated at high flux. A second evaporation mode using dispenser currents about 3-4.5 A will be denoted as the "cool" evaporation mode. The quantity of $\mathrm{Rb}$ adsorbed on the surface of the layered crystals was carefully calibrated by surface photovoltage measurements ${ }^{28,29}$ and scanning tunneling microscopy. By adjusting the evaporation time similar amounts of $\mathrm{Rb}$ atoms were adsorbed in both modes.

For the cool evaporation mode employing a relatively low flux (about one-tenth of the hot case) it is likely that a random adsorption of $\mathrm{Rb}$ atoms on the surface is achieved. Due to a low activation barrier for surface diffusion ${ }^{30,23}$ these atoms are highly mobile on the surface and thus cannot be visualized in STM images taken at room temperature.

For the hot $\mathrm{Rb}$ exposure a completely different adsorption behavior is observed. Instead of a random distribution of $\mathrm{Rb}$ atoms, networks of $\mathrm{Rb}$ containing nanowires are obtained ${ }^{23}$ (see Fig. 2). Depending on the time of Rb exposure open and closed networks can be generated. While an open network [Fig. 2(a)] shows a number of nanowires with open ends, closed networks [Fig. 2(b)] almost exclusively exhibit complete meshes. The nanowires start to grow along extremely weak irregularities (folds) of the surface layer, which can eventually be found on otherwise extremely flat surfaces of any TMDC grown by CVT. ${ }^{31}$ These folds separate slightly tilted $\left(<0.02^{\circ}\right)$ surface areas. ${ }^{23}$ A wire end induces stress along the fold, which leads to a crack in the topmost surface layer. It should be noted here that these cracks were not found on the clean substrate. Acting like a condensing gas, highly diffusing $\mathrm{Rb}$ atoms at room temperature will be trapped by the cracks after hopping over the Schwoebel barrier at the edges of the cracks and driving the crack further along the fold. Diffusion length along the cracks is apparently large since no wire fragments can be observed. This growth mode of the nanowires is schematically depicted in Fig. 3(a). A side view of the topmost three layers is shown in Fig. 3(b). Rb wires separate areas in the topmost surface layer thereby introducing stress in these layer fragments (indicated by the corrugation). Stopping growth in an intermediate state thus results in an open network. Further growth finally leads to a closed network of $\mathrm{Rb}$ nanowires.

It is important to note that the rubidium contained in the wires stays for more than 1 month on the surface. A tardy in situ intercalation can therefore be excluded. This behavior differs from that of other adsorbates on $1 T-\mathrm{TaS}_{2}$, e.g., for $\mathrm{Na}$ or $\mathrm{Cu},{ }^{20,32}$ it is believed that a rapid intercalation occurs directly through the layers.

\section{RESULTS AND DISCUSSION}

\section{A. CDW superstructures}

The clean $1 T-\mathrm{TaS}_{2}$ surface exhibits the well known $(\sqrt{13} \times \sqrt{13}) R 13.9^{\circ}$ superstructure according to the nearly 

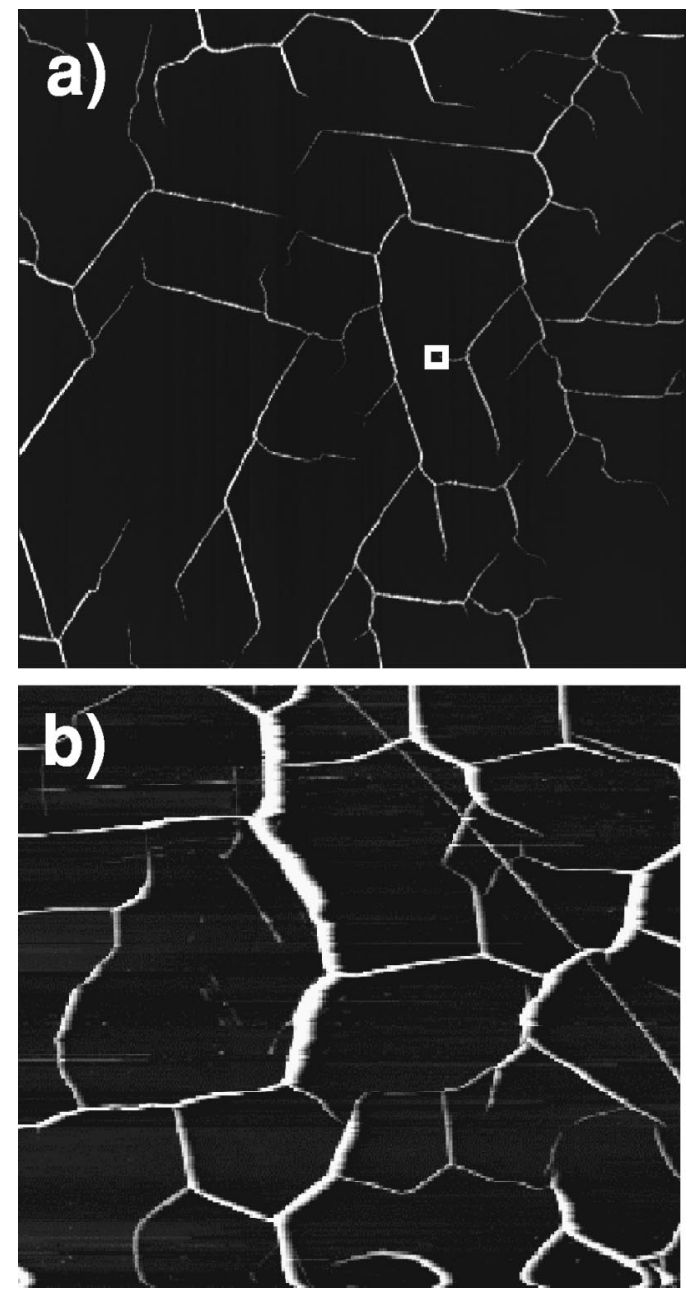

FIG. 2. STM empty state images (bias $0.7 \mathrm{~V}, I_{T}=200 \mathrm{pA}$ ) of Rb nanowire covered $1 T-\mathrm{TaS}_{2}$ surfaces. (a) Open nanowire network obtained after $3 \mathrm{~min} \mathrm{Rb}$ exposure. The white square is magnified in Fig. 4 below. (b) Closed network obtained after 4.5 min Rb exposure.

commensurate room temperature CDW phase. The hexagonal array of CDW domains is clearly evident in the STM image of Fig. 1(b). The corresponding LEED pattern nicely shows the CDW superstructure. Upon Rb adsorption this CDW superstructure is suppressed and, depending on the type of $\mathrm{Rb}$ exposure, a variety of new CDW superstructures are formed.

Upon hot evaporation of $\mathrm{Rb}$ with an exposure time of 3 min resulting in an open network of $\mathrm{Rb}$ nanowires on the surface, the most dramatic changes of the CDW superstructure are observed. The corresponding LEED pattern shown in Fig. 4(d) reveals a large number of superstructure LEED spots. It should be noted here that similar LEED patterns were obtained after electrochemical intercalation of $\mathrm{Na}$ or $\mathrm{N}_{2} \mathrm{H}_{4}$ in $\mathrm{TaS}_{2},{ }^{20,19}$ and were assigned to a $\sqrt{7} / 8 \times \sqrt{7} / 8$ threedomain CDW superstructure. Following the notation by Wood, however, this superstructure should be described as $c(2 \sqrt{3} \times 4)$ rect. ${ }^{33,34}$ The occurrence of three CDW domains, however, requires a reduction of surface symmetry. Since LEED investigations cannot give detailed insight into the
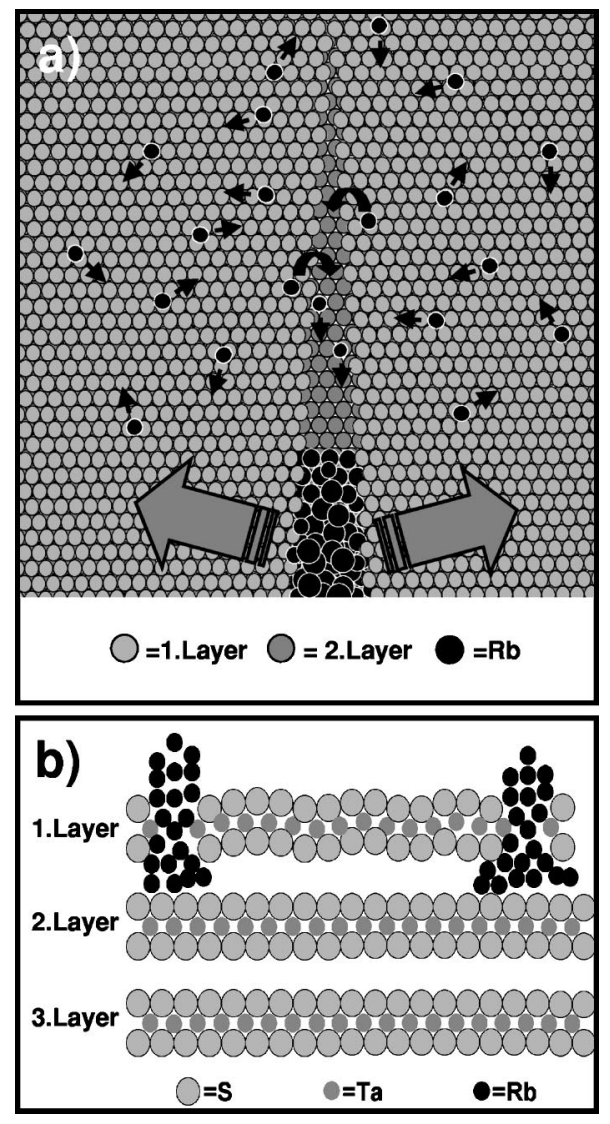

FIG. 3. Schematic of $\mathrm{Rb}$ nanowires grown on layered materials. (a) Top view during growth. The big arrows beside the "wire" indicate stress induced by the nanowire. (b) Side view of the top three layers with grown wires. The corrugation of the top layer is due to the induced stress.

structure of each of the three domains STM measurements have been performed.

Figure 4(a) shows a $120 \mathrm{~nm} \times 123 \mathrm{~nm}$ sized STM picture. This enlargement of Fig. 2(a) shows a crack pattern, which is induced by stress at the end of a wire. ${ }^{23}$ Such a pattern has never been found on a clean sample surface. The network of crack lines and wires divides the sample into single domains. In each domain only one type of CDW can be found. Figure 4(a) shows an enlargement of a wire end, where three (I,II, III) CDW domains were found, which are rotated by $60^{\circ}$ to each other. The LEED pattern in Fig. 4(d) can be composed by combining the power spectra of the three individual zones, which are marked by I, II, and III in Fig. 4(c). In this way it is possible to assign each spot to one of the three domains and assign the LEED pattern to three identical superstructures that are rotated by $60^{\circ}$ to each other.

It should be noted here that the $c(2 \sqrt{3} \times 4)$ rect. superstructure, although misleadingly assigned to $\sqrt{7} / 8 \times \sqrt{7} / 8$ has also been observed in electrochemical intercalation studies of $\mathrm{N}_{2} \mathrm{H}_{4}$ in $\mathrm{TaS}_{2}{ }^{19}$ The occurrence of a twofold symmetry in the CDW superstructure, however, was explained as being induced by the twofold symmetry of the intercalated molecule. Since $\mathrm{Rb}$ is evaporated atom by atom and thus has full spherical symmetry it cannot explain the superstructure observed here. The origin of the reduction of surface symmetry 


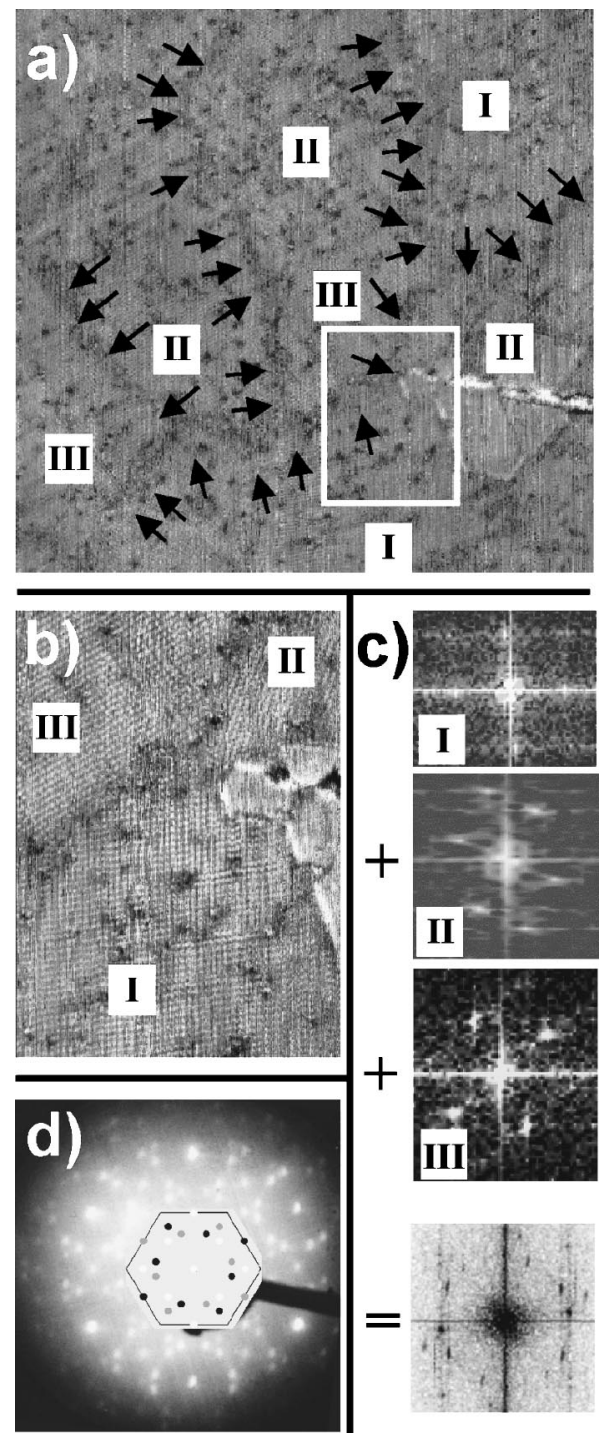

FIG. 4. (a) and (b) Magnification of the STM image of Fig. 2(a) (open $\mathrm{Rb}$ nanowire network on $1 T-\mathrm{TaS}_{2}$ ) in the vicinity of an end of a nanowire. Folds separating different CDW domains assigned as I, II, and III are evident as dark gray lines. (c) Power spectra of different CDW domains and sum compared to LEED pattern in (d), which can be identified as $c(2 \sqrt{3} \times 4)$ rect. superstructure.

may be found in the type of stress induced by the nanowires. As schematically depicted in Fig. 3(a) parallel wires induce compressive stress perpendicular to the direction of the wires. For an open network this type of stress dominates, giving rise to three domains of lower symmetry. So it is likely that the surrounding wires and/or the crack lines determine the alignment of the CDW's by providing nonuniform stress. It may thus be possible that strain fields induced by nonuniform intercalation can also explain the CDW phases found in earlier intercalation studies.

After $4.5 \mathrm{~min} \mathrm{Rb}$ exposure in the hot evaporation mode a closed network of $\mathrm{Rb}$ nanowires is found on the surface [see Fig. 2(b)]. The corresponding LEED pattern exhibiting a (single domain) $3 \times 3$ superstructure is shown in Fig. 5(c). The number of open ended wires is drastically reduced in a a)
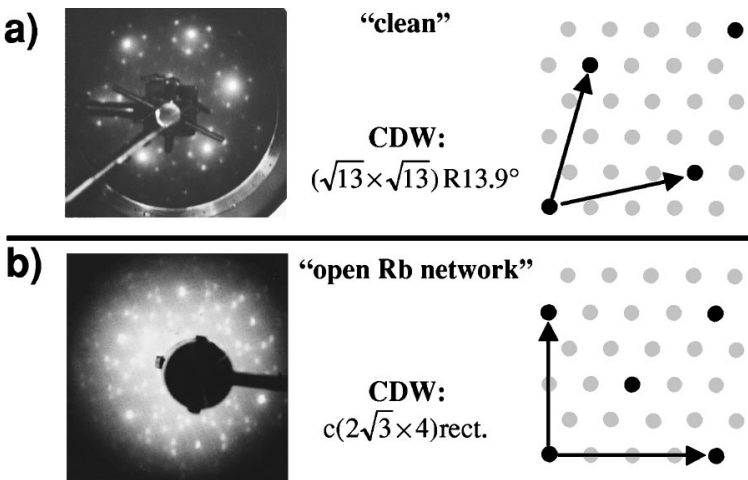

"open Rb network"

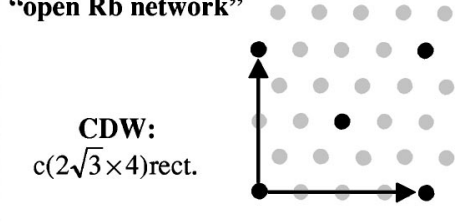

c)

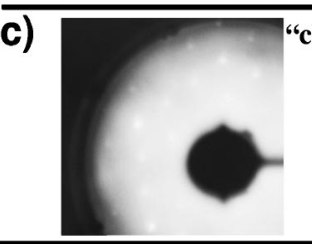

"closed Rb network"

d)

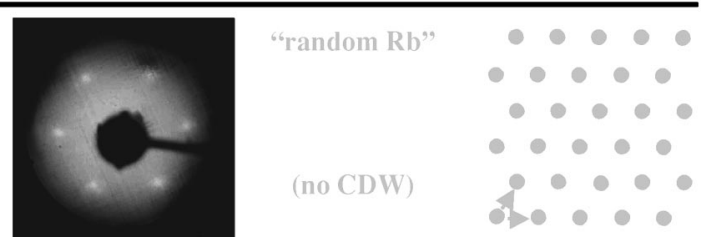

FIG. 5. LEED patterns and CDW superstructures for clean and $\mathrm{Rb}$ exposed $1 T-\mathrm{TaS}_{2}$. (a) commensurate CDW structure of the clean crystal; (b) one of the three $c(2 \sqrt{3} \times 4)$ rect. domains found after the hot evaporation of $3 \mathrm{~min}$; (c) $3 \times 3$ superstructure observed after the hot evaporation for a longer time of $4.5 \mathrm{~min}$; (d) no CDW superstructure obtained after the cool evaporation.

closed network. Consequently the uniaxial stress from parallel open ended wires is replaced by hydrostatic pressure from wires closed around single meshes. In this case a reduction of symmetry is less likely than a homogeneous shrinking of the substrate lattice in the first layer. Therefore a threedomain CDW superstructure is not expected here.

In the cool evaporation mode the charge density wave pattern completely vanishes and the LEED image shows only a $1 \times 1$ pattern representing the unit cell of $1 T-\mathrm{TaS}_{2}$. The varieties of CDW phases observed on clean and $\mathrm{Rb}$ exposed $1 T-\mathrm{TaS}_{2}$ surfaces are summarized in Fig. 5. Since in intercalation studies even more different thermodynamic phases of $\mathrm{TaS}_{2}$ are found, ${ }^{20}$ it is likely that additional phases may exist at different wire coverages.

\section{B. Electronic structure}

The geometric superstructures of the various charge density wave phases of clean and $\mathrm{Rb}$ exposed $1 T-\mathrm{TaS}_{2}$ are intimately related to characteristic features in their corresponding electronic structures. Angle resolved photoemission spectroscopy probing the occupied electronic states is a powerful tool to evaluate these variations.

In Fig. 6 we show angle resolved photoemission spectra taken in normal electron emission at $21.2 \mathrm{eV}$ photon energy. The spectrum at the top represents the clean $1 T-\mathrm{TaS}_{2}$ surface, revealing the nearly commensurate $\mathrm{CDW}$ phase at room temperature. The electron emissions are characterized 


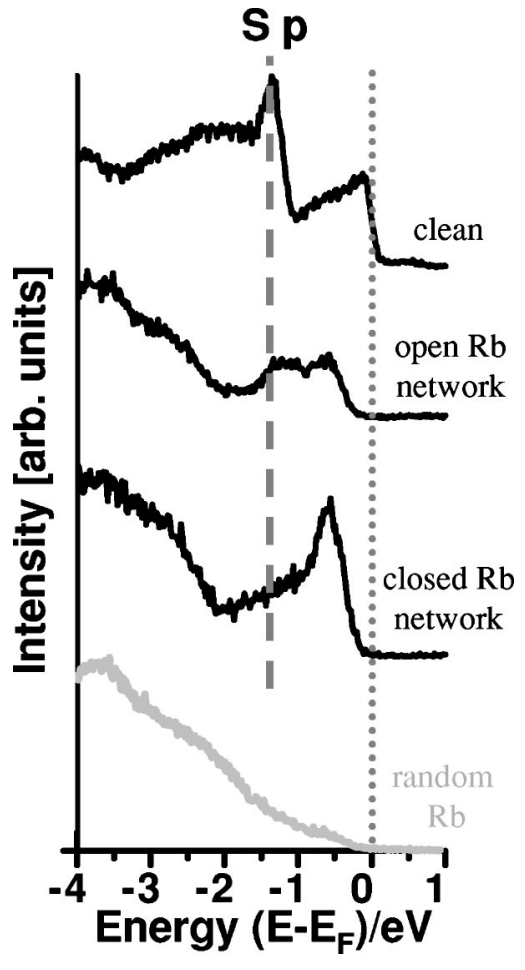

FIG. 6. Angle resolved photoemission valence band spectra of clean and $\mathrm{Rb}$ exposed $1 T-\mathrm{TaS}_{2}$ taken in normal electron emission at $21.22 \mathrm{eV}$ photon energy.

by Ta $5 d$ valence bands at binding energies between the Fermi level and $-1.5 \mathrm{eV}$. The features below $-1 \mathrm{eV}$ binding energy originate from $p$-derived sulfur states. Upon $\mathrm{Rb}$ exposure and associated growth of nanowires, dramatic changes are evident. For open and closed network geometries the electron density at the Fermi level is suppressed, thereby opening a gap between occupied and unoccupied electronic states.

It should be noted here that this metal-insulator transition is driven by growth of nanowires on the surface of the layered material. It has been shown that the growth of the nanowires is accompanied by a decrease of $k$ dispersion of electronic states perpendicular to the layers. ${ }^{35,36}$ This gives evidence for an electronic decoupling of the first layer from the bulk. The $k$ dispersion parallel to the layers, however, is preserved. The energy gap in the insulating state amounts to roughly $0.4 \mathrm{eV}$ in the occupied part, which is more than one order of magnitude larger than the gap observed in the commensurate phase of clean $1 T-\mathrm{TaS}_{2}$ below $180 \mathrm{~K} .^{3,13}$ More drastically, it appears that the highest-energy $p$-orbitalderived structure (about $1.5 \mathrm{eV}$ below the Fermi level) vanishes completely (see dashed line). Such behavior which is also observed in intercalation studies ${ }^{22}$ is due to a decreasing overlap of $p$ orbitals when the layer separation is increased. This also corroborates the nanowire induced elevation of the first layer.

A spectrum of a $\mathrm{Rb}$ exposed surface employing the cool evaporation mode is depicted at the bottom of Fig. 6. The spectrum is characterized by suppressed valence band emissions and a high low-energy background, which is typical for a contaminated surface.

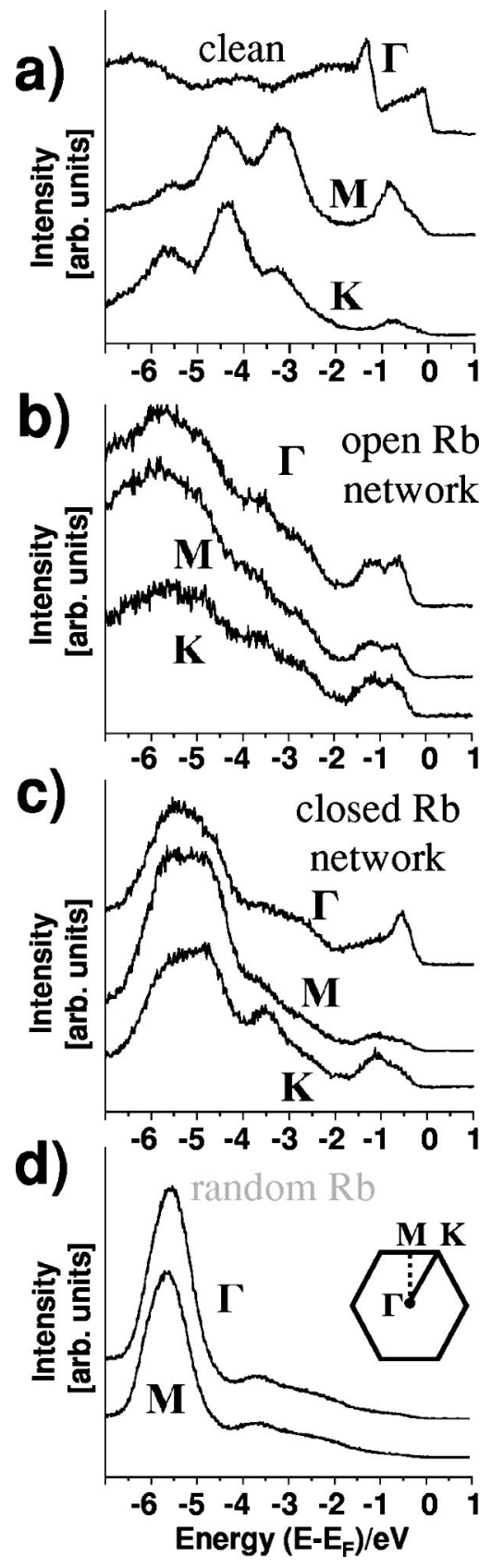

FIG. 7. Angle resolved photoemission valence band spectra of clean and $\mathrm{Rb}$ exposed $1 T-\mathrm{TaS}_{2}$ taken at the high-symmetry points $\Gamma, M$, and $K$ of the Brillouin zone (see inset). Photon energy was $21.22 \mathrm{eV}$.

The dispersion of electronic states parallel to the layers is also affected by the different CDW superstructures. In Fig. 7 we show angle resolved photoemission spectra taken at the high-symmetry points $\Gamma, M$, and $K$ (see the Brillouin zone in the inset) for clean and $\mathrm{Rb}$ exposed $1 T-\mathrm{TaS}_{2}$. The clean sample in Fig. 7(a) exhibits clear differences between the high-symmetry points. For an open $\mathrm{Rb}$ network the spectra taken at different high-symmetry points look very similar. In the closed network case the dispersion appears again [see Fig. 7(c)]. Since every closed network starts as an open network, the return of the dispersion appears surprising at first. 


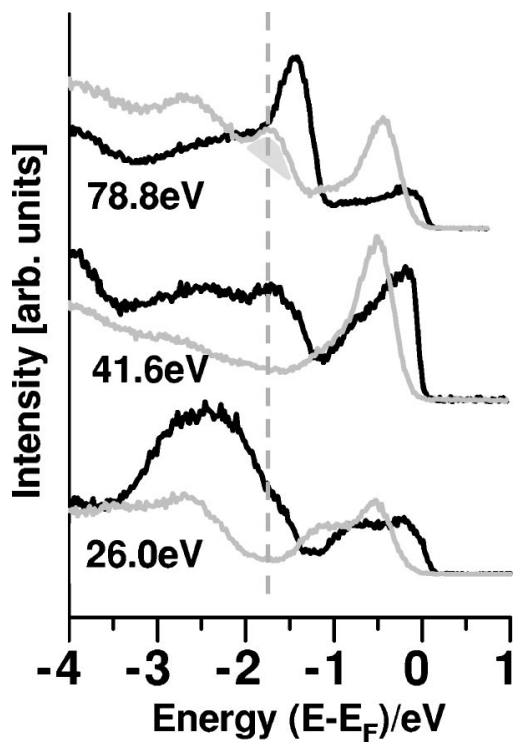

FIG. 8. Valence band spectra obtained from clean $1 T-\mathrm{TaS}_{2}$ (black) and closed $\mathrm{Rb}$ network covered surfaces (gray) taken at higher photon energies. The suppressed sulfur $p$ state structure (hatched) reappears in the $78.8 \mathrm{eV}$ spectrum due to enhanced bulk sensitivity (marked by dashed line).

However, for the open $\mathrm{Rb}$ nanowire network case there are three $c(2 \sqrt{3} \times 4)$ rect. CDW domains (each being rotated by $60^{\circ}$ ) present on the surface. Since photoemisssion integrates over these domains the dispersion appears to be smeared out. For a closed $\mathrm{Rb}$ nanowire network, in contrast, only one 3 $\times 3$ domain is observed and stronger dispersion of electronic states parallel to the layers is restored. In contrast to the network covered samples, randomly adsorbed $\mathrm{Rb}$ on the surface leads to vanishing dispersion and a huge inelastic background. Such features are typical for a contaminated surface in photoemission. It is likely that the disordered $\mathrm{Rb}$ atoms strongly scatter the photoelectrons.

The surface sensitivity of photoemission can be tuned by adjusting the kinetic energy of the detected electrons. Consequently, by taking photoemission valence band spectra at different photon energies different layers perpendicular to the surface can be probed. While for kinetic energies between 20 and $40 \mathrm{eV}$ the surface sensitivity is highest and mainly the uppermost surface layer is probed, for higher energies electrons excited in the second layer can also escape through the surface.

A series of normal emission photoemission spectra taken at 26, 41.6, and $78.8 \mathrm{eV}$ photon energy on clean (black curves) and $\mathrm{Rb}$ exposed (closed network, gray curves) $1 T-\mathrm{TaS}_{2}$ surfaces is shown in Fig. 8. At $41.6 \mathrm{eV}$ photon energy the characteristic sulfur $p$ states between -1 and $-3 \mathrm{eV}$ (black curve) are suppressed after nanowire formation (gray curve), constituting the $p-d$ gap. At higher photon energies the escape depth for photoelectrons reaches the second layer. As this layer represents the untouched substrate, photoemission spectra at these photon energies must be a superposition of the electronic spectra of the two dimensional first layer on the bulk substrate spectra. This is what

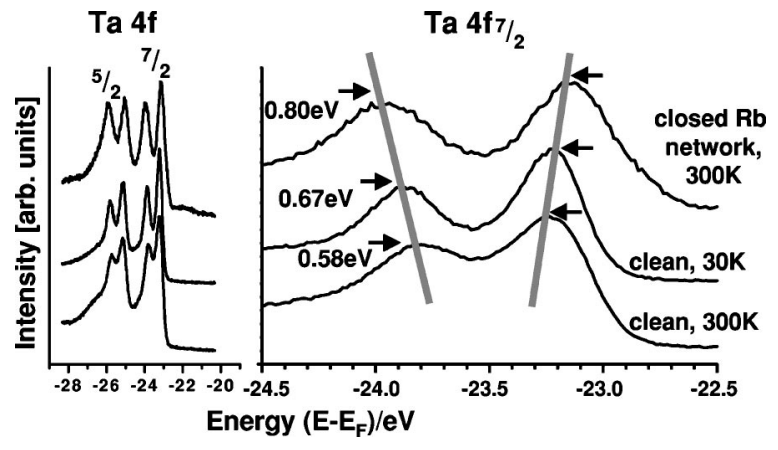

FIG. 9. Ta $4 f$ core level spectra $(h \nu=78 \mathrm{eV})$ for clean (lower and middle spectra taken at $300 \mathrm{~K}$ and $30 \mathrm{~K}$, respectively) and $\mathrm{Rb}$ nanowire network covered $1 T-\mathrm{TaS}_{2}$ (top spectrum). Right: magnification of the Ta $4 f_{7 / 2}$ level. Solid lines indicate the CDW induced splittings. Spectra taken with a total energy resolution of $0.03 \mathrm{eV}$.

can be observed in Fig. 5: In the $p-d$ gap parts of the $p$-derived sulfur emission are found again, indicating that the following layers are unaffected by the changed CDW phase of the topmost layer.

CDW's that are associated with a periodic lattice distortion are also evident in Ta $4 f$ core level spectra. Figure 9 shows the Ta $4 f \frac{5}{2}$ and $\frac{7}{2}$ doublets of the clean sample measured at $300 \mathrm{~K}$ and $30 \mathrm{~K}$, respectively, and a spectrum of a sample covered with a network of $\mathrm{Rb}$ nanowires. The Ta $4 f$ core levels are additionally split due to inequivalent lattice positions of the $\mathrm{Ta}$ atoms in the charge density wave phase. ${ }^{37-39}$ The increased splitting of the core levels observed at lower temperature (see Fig. 9) is well known and induced by the phase transition from the $\mathrm{NC}$ to the $\mathrm{C}$ phase of clean $1 T-\mathrm{TaS}_{2}$. It represents the energy gain of the system due to the CDW lock-in. For the $\mathrm{Rb}$ nanowire network covered sample an even larger splitting is observed. A more detailed analysis, however, appears to be difficult because the core levels may be composed from an overlap of the first $3 \times 3$ superstructured (nanowire) layer and the second layer, which is believed to still be maintained in the NC phase.

\section{SUMMARY AND CONCLUSIONS}

We have investigated the structural and electronic properties of the clean and $\mathrm{Rb}$ exposed charge density wave material $1 T-\mathrm{TaS}_{2}$. It is found that $\mathrm{Rb}$ adsorbs on the surface of the layered material and does not intercalate into the van der Waals gaps between the layers. Depending on the evaporation mode $\mathrm{Rb}$ adsorbs randomly or forms different networks of nanowires. Upon $\mathrm{Rb}$ exposure the charge density wave superstructure changes from $(\sqrt{13} \times \sqrt{13}) R 13.9^{\circ}$ for the clean surface to $c(2 \sqrt{3} \times 4)$ rect. or $3 \times 3$ depending on the type of network. The transformation of the superstructures appears to be driven by stress that is induced by the nanowires. This contrasts with earlier intercalation studies using other materials, where it was concluded that the changes of the superstructures were induced by the symmetry or periodicity of the intercalated species. In the electronic structure the nanowire induced CDW changes are associated with a metalinsulator transition, which reflects the energy gain due to 
formation of the superstructures. Photoemission measurements using different photon energies and surface sensitivities give evidence that the nanowire induced CDW is two dimensional and appears to be located in the first layer only. Due to the periodic lattice distortions Ta $4 f$ core levels are split according to the different Ta positions.

\section{ACKNOWLEDGMENTS}

The authors would like to thank J. Crawack, C. Pettenkofer, H. I. Starnberg, and W. Jaegermann for fruitful discussions. This research was supported by the BMBF, F.R. Germany (Project No. 05 SB8 FKB).
${ }^{1}$ F. DiSalvo, J. Wilson, B. Bagley, and J. Waszczak, Phys. Rev. B 12, 2220 (1975).

${ }^{2}$ G. K. Wertheim, F. J. DiSalvo, and S. Chiang, Phys. Rev. B 13, 5476 (1976).

${ }^{3}$ R. Manzke, O. Anderson, and M. Skibowski, J. Phys. C 21, 2399 (1988).

${ }^{4}$ B. Burk, R. E. Thomson, A. Zettl, and John Clarke, Phys. Rev. Lett. 66, 3040 (1991).

${ }^{5}$ F. Zwick, H. Berger, I. Vobornik, G. Margaritondo, L. Forro, C. Beeli, M. Onellion, G. Panaccione, A. Taleb-Ibrahimi, and M. Grioni, Phys. Rev. Lett. 81, 1058 (1998).

${ }^{6}$ Th. Pillo, J. Hayoz, H. Berger, R. Fasel, L. Schlapbach, and P. Aebi, Phys. Rev. B 62, 4277 (2000).

${ }^{7}$ J. E. Hirsch and D. J. Scalapino, Phys. Rev. Lett. 56, 2732 (1986).

${ }^{8}$ D. M. News, H. R. Krishnamurthy, P. C. Pattnaik, C. C. Tsuei, and C. L. Cane, Phys. Rev. Lett. 69, 1264 (1992).

${ }^{9}$ V. Vescoli, L. Degiorgi, H. Berger, and L. Forro, Phys. Rev. Lett. 81, 453 (1998).

${ }^{10}$ P. Fazkas and E. Tosatti, Philos. Mag. B 39, 229 (1979).

${ }^{11}$ R. Manzke, T. Buslaps, B. Pfalzgraf, M. Skibowski, and O. Anderson, Europhys. Lett. 8, 195 (1989).

${ }^{12}$ B. Dardel, M. Grioni, D. Malterre, P. Weibel, Y. Baer, and F. Levy, Phys. Rev. B 46, 7407 (1992).

${ }^{13}$ M. Boehme, L. Kipp, and M. Skibowski, Solid State Commun. 112, 101 (1999).

${ }^{14}$ K. Motizuki, Structural Phase Transitions in Layered Transition Metal Compounds (Reidel, Dordetrecht, 1986).

${ }^{15}$ R. Peierls, Ann. Phys. (Leipzig) 5(4), 2 (1930).

${ }^{16}$ G. Grüner, Density Waves in Solids (Addison-Wesley, Reading, MA, 1994).

${ }^{17}$ W. Y. Liang, in Intercalation in Layered Materials, Vol. 148 of NATO Advanced Study Institute, Series B: Physics, edited by M. S. Dresselhaus (Plenum, New York, 1986).

${ }^{18}$ R. Friend and A. Yoffe, Advances in Physics (Taylor and Francis, London, 1987), Vol. 36.

${ }^{19}$ G. J. Tatlock and J. V. Acrivos, Philos. Mag. B 38, 81 (1978).

${ }^{20}$ C. Pettenkofer and W. Jaegermann, Phys. Rev. B 50, 8816 (1994)

${ }^{21}$ I. Ekvall, H. E. Brauer, E. Wahlström, and H. Olin, Phys. Rev. B 59, 7751 (1999).
${ }^{22}$ H. I. Starnberg, H. E. Brauer, L. J. Holleboom, and H. P. Hughes, Phys. Rev. Lett. 70, 3111 (1993).

${ }^{23}$ R. Adelung, L. Kipp, J. Brandt, L. Tarcak, M. Traving, C. Kreis, and M. Skibowski, Appl. Phys. Lett. 74, 3053 (1999).

${ }^{24}$ Due to the shape of the wires (they are roughly as wide as high) the most part of the $\mathrm{Rb}$ is "hidden" in the bulk of the wires. One complete monolayer of $\mathrm{Rb}$ will thus lead to an effective wire coverage of the surface of approximately $4 \%$.

${ }^{25}$ The STM is a standard Omicron micro STM, which has been improved by a highly efficient eddy current vibration damping system.

${ }^{26}$ K. Rossnagel, L. Kipp, M. Skibowski, and S. Harm, Nucl. Instrum. Methods (to be published).

${ }^{27}$ SAES Getter Sources S.p.A., Milan, Italy.

${ }^{28}$ L. Kipp, R. Adelung, N. Trares-Wrobel, and M. Skibowski, Appl. Phys. Lett. 74, 1836 (1999).

${ }^{29}$ M. Boehme, R. Adelung, M. Traving, L. Kipp, and M. Skibowski, Appl. Surf. Sci. 123/124, 91 (1998).

${ }^{30}$ C. Ramirez and W. Schattke, Surf. Sci. (to be published).

${ }^{31}$ C. Dieker, R. Adelung, L. Kipp, M. Skibowski, and W. Jäger (unpublished).

${ }^{32}$ C. Pettenkofer, W. Jaegermann, and B. A. Parkinson, Surf. Sci. 251/252, 583 (1991).

${ }^{33}$ The NIST Surface Structure Database (SSD) represents a joint effort of the Standard Reference Data Group at the National Institute of Standards and Technology (NIST), Washington, DC, and of three main investigators, P. R. Watson (OSU Corvallis), M. A. Van Hove (UC Berkeley and LBL), and K. Hermann (Fritz-Haber-Institut Berlin).

${ }^{34}$ R. F. Lin, G. S. Blackman, M. A. Van Hove, and G. A. Somorjai, Acta Crystallogr., Sect. B: Struct. Sci. 43, 368 (1987).

${ }^{35}$ R. Adelung, J. Brandt, K. Rossnagel, O. Seifarth, L. Kipp, M. Skibowski, C. Ramirez, T. Strasser, and W. Schattke, Phys. Rev. Lett. 86, 7303 (2001).

${ }^{36}$ R. Adelung, J. Brandt, L. Tarcak, L. Kipp, and M. Skibowski, Appl. Surf. Sci. 162-163, 666 (2000).

${ }^{37}$ R. A. Pollak, D. E. Eastman, F. J. Himpsel, P. Heimann, and B. Reihl, Phys. Rev. B 24, 7435 (1981).

${ }^{38}$ G. K. Wertheim, F. J. DiSalvo, and S. Chiang, Phys. Lett. 54A, 3046 (1975).

${ }^{39}$ H. P. Hughes and R. Pollak, Philos. Mag. 34, 1025 (1976). 